\title{
ON THE GROWTH OF FUNDAMENTAL GROUPS OF NONPOSITIVE CURVATURE MANIFOLDS
}

\begin{abstract}
YI-HU YANG
Milnor's classic result that the fundamental group of a compact Riemannian manifold of negative sectional curvature has exponential growth is generalised to the case of negative Ricci curvature and non-positive sectional curvature.
\end{abstract}

\section{INTRODUCTION AND MAIN RESULTS}

In the paper [1], Milnor proved the following:

THEOREM. If $M$ is a compact Riemannian manifold with sectional curvature less than zero, then the growth function of the fundamental group $\pi_{1} M$ is at least exponential:

$$
r(s) \geqslant a^{s}
$$

for some constant $a>1$, which depends on the particular finite set of generators for $\pi_{1} M$ which is used to define $r(s)$.

Generally, $r(s)$ is defined as follows. Let $G$ be a finitely generated group and $g_{1}, \cdots, g_{p}$ a specified set of generators for $\pi_{1} M$. Then $r(s)$ associated with $G$ and $g_{1}, \cdots, g_{p}$ is the number of distinct group elements which can be expressed as the words of length $\leqslant s$ in the specified generators and their inverses. In addition, Milnor also proved that whether $r(s)$ is of exponential growth does not depends on the particular finite set of generators for $\pi_{1} M$.

In the paper, Milnor conjectured that the curvature condition in the Theorem can be weakened to negative Ricci curvature. The purpose of this note is to confirm this conjecture. Namely, we shall show the following:

THEOREM A. Let $N$ is a compact Riemannian manifold with nonpositive sectional curvature and negative Ricci curvature. Then, the growth function of the fundamental group $\pi_{1} N$ is at least exponential.

The key point of our proof is to give a lower bound estimate for the volume of the geodesic balls of the universal covering for $N$. This is the following:

Received 4th January, 1996

This work supported in part by Chinese Post-doctoral Science Foundation. The author would like to express his thanks to the Referee.

Copyright Clearance Centre, Inc. Serial-fee code: 0004-9729/96 $\$$ A2.00+0.00. 
Theorem B. Let $M$ be a $n$-dimensional Cartan-Hadamard manifold, with the Ricci curvature of $M \leqslant-(n-1) b^{2}, b>0$. Then for any fixed $\varepsilon>0$, there exsits a positive constant $c(\varepsilon, n)$ such that when $r \geqslant \varepsilon$, one has

$$
V_{x_{0}}(r) \geqslant c(\varepsilon, n) e^{b r}
$$

where $V_{x_{0}}(r)$ is the volume of the geodesic ball centred at $x_{0} \in M$ with radius $r$.

\section{Proof of Theorem A}

The idea of the proof is the same as that of Milnor. For completeness, we write it down. Let $M$ be the universal covering space of $N$. Since $N$ is of negative Ricci curvature and nonpositive sectional curvature, then $M$ is Cartan-Hadamard manifold and there is a positive constant, say $b$, such that the Ricci curvature of $M \leqslant-(n-1) b^{2}$. Let $d$ be the diameter of $N$, then $P: B_{x_{0}}(d)(\subset M) \rightarrow N$ is surjective. Here $P$ is the covering map from $M$ to $N$, and $B_{x_{0}}(d)$ is the geodesic ball in $M$ centred at $x_{0}$ with radius $d$. Denote $B_{x_{0}}(d)$ by $B$ in the following. Let $F=\left\{g \in \pi_{1} N \mid g B \cap B \neq \emptyset\right\}$ and $\left.\mu=\min _{\operatorname{dist}}(B, g B) \mid g B \cap B=\emptyset, g \in \pi_{1} N\right\}$. We claim $F$ generates $\pi_{1} N$. Let $\operatorname{dist}_{M}\left(x_{0}, g B\right)<\mu t+d$. Take $y \in g B$ with $\operatorname{dist}_{M}\left(x_{0}, y\right)<\mu t+d$. Let $\gamma:\left[0, \operatorname{dist}_{M}\left(x_{0}, y\right)\right] \rightarrow M$ be the geodesic joining $x_{0}$ and $y$. In turn, taking $y_{1}, \cdots, y_{t}, y_{t+1}=y$ on $\gamma\left(\left[0, \operatorname{dist}_{M}\left(x_{0}, y\right)\right]\right)$ satisfying $\operatorname{dist}_{M}\left(x_{0}, y_{1}\right)<d$ and $\operatorname{dist}_{M}\left(y_{i}, y_{i+1}\right)<\mu$ for arbitrary $i \in\{1,2, \cdots, t\}$, then there exist $f_{i} \in \pi_{1} N(i=1, \cdots, t+1)$ such that $y_{i} \in f_{i} B, f_{1}=e$ and $f_{t+1}=g$. Thus, $\operatorname{dist}_{M}\left(f_{i} B, f_{i+1} B\right)<\mu$. By the definitions of $\mu$ and $F$, we have $f_{t}^{-1} f_{i+1} \in F$. It is obvious that $g=f_{1}^{-1} f_{2} f_{2}^{-1} f_{3} \cdots f_{t}^{-1} f_{t+1}$, that is, $g$ is generated by $F$. On the other hand, $F$ is also a finite set of $\pi_{1} N$, therefore $\pi_{1} N$ is finitely generated. In addition, we also obtain that $g$ can expressed in at most $t$ elements in $F$ assuming $\operatorname{dist}_{M}\left(x_{0}, g B\right)<\mu t+d$.

Let $A=\left\{g \in \pi_{1} N \mid g B \cap B_{x_{0}}(\mu t+d) \neq \emptyset\right\}$. The above conclusion shows any element in $A$ can be expressed in at most $t$ elements in $F$. So $r(t) \geqslant$ the number of elements in $A$, denoted by $|A|$. On the other hand, $\{g B \mid g \in A\}$ covers $B_{x_{0}}(\mu t+d)$, then $|A| V_{x_{0}}(d) \geqslant V_{x_{0}}(\mu t+d)$. Using Theorem $\mathrm{B}$, we have

$$
r(t) \geqslant \frac{c(\varepsilon, n) e^{b(\mu t+d)}}{V_{z_{0}}(d)} \geqslant c^{\prime} a^{t},(a>1) .
$$

The proof of Theorem A is completed.

\section{Proof of Theorem B}

We shall use the following Laplacian comparision theorem from [2]. (For a simpler proof, see [3].) 
Theorem C. Let $M, \widetilde{M}$ be two $n$-dimensional Cartan-Hadamard manifolds, $x_{0} \in M, \widetilde{x_{0}} \in \widetilde{M}, \rho(x)=\operatorname{dist}_{M}\left(x, x_{0}\right), \tilde{\rho}(\widetilde{x})=\operatorname{dist}_{\widetilde{M}}\left(\widetilde{x_{1}}, \widetilde{x_{0}}\right)$. Assume

$$
\operatorname{Ric}\left(\frac{\partial}{\partial \rho}, \frac{\partial}{\partial \rho}\right)(x) \leqslant \widetilde{\operatorname{Ric}}\left(\frac{\partial}{\partial \widetilde{\rho}}, \frac{\partial}{\partial \tilde{\rho}}\right)(\widetilde{x})
$$

for $\rho(x)=\tilde{\rho}(\widetilde{x})$, where Ric, $\widetilde{R i c}$ are the Ricci curvatures of $M$ and $\widetilde{M}$ respectively. Then

$$
\Delta \rho \geqslant \frac{1}{n-1} \tilde{\Delta} \tilde{\rho}
$$

where $\Delta, \widetilde{\Delta}$ are the Laplace-Beltrami operators of $M$ and $\widetilde{M}$ respectively. In particular, if $\operatorname{Ric}\left(\frac{\partial}{\partial \rho}, \frac{\partial}{\partial \rho}\right) \leqslant-(n-1) b^{2}$, one has

$$
\Delta \rho \geqslant b \operatorname{coth}(b r)
$$

Let $x_{0} \in M$, a fixed point, with the distance function at $x_{0}$, denoted by $\rho$. Let $X \in T_{x_{0}} M$ with $|X|=1$, and consider the geodesic $\gamma(t)=\exp _{x_{0}} t X$. Define the function $\phi:[0, \infty) \rightarrow R$ as follows,

$$
t \rightarrow \phi(t)=\left|\frac{\exp p_{x_{0}}^{*} \Omega}{\widetilde{\Omega}}\right|
$$

where $\exp p_{x_{0}}^{*}$ is the adjoint operator of $\exp _{x_{0}}, \Omega$ and $\widetilde{\Omega}$ are the volume elements of $M$ and $T_{x_{0}} M$ at $\exp _{x_{0}} t X$ and $t X$ respectively. Similarly, we can define $\phi: T_{x_{0}} M \rightarrow R$ as follows,

$$
X \rightarrow \phi(X)=\left|\frac{e x p_{x_{0}}^{*} \Omega}{\widetilde{\Omega}}\right|,
$$

where $\Omega$ and $\widetilde{\Omega}$ are the volume elements of $M$ and $T_{x_{0}} M$ at $\exp _{x_{0}} X$ and $X$ respectively. Let $J_{1}, \cdots, J_{n-1}$ be linearly independent normal Jacobi fields along the geodesic $\gamma(t)$ with $J_{i}(0)=0, i=1, \cdots, n-1$. Then, Jacobi field theory tells us

$$
\phi(t)=\frac{\left|J_{1}(t) \wedge J_{2}(t) \wedge \cdots \wedge J_{n-1}(t)\right|}{t^{n-1}\left|J_{1}^{\prime}(0) \wedge J_{2}^{\prime}(0) \wedge \cdots \wedge J_{n-1}^{\prime}(0)\right|},
$$

where $\left|A_{1} \wedge A_{2} \wedge \cdots \wedge A_{n}\right|=\sqrt{\operatorname{det}\left(\left\langle A_{i}, A_{j}\right\rangle\right)}$. A direct computation shows

$$
\frac{\phi^{\prime}(t)}{\phi(t)}=\left(\Delta \rho-\frac{n-1}{\rho}\right) \circ \gamma(t) \text {. }
$$

Here, we have used the fact $(\Delta \rho) \circ \gamma(t)=\sum_{i=1}^{n-1}\left\langle J_{i}^{\prime}(t), J_{i}(t)\right\rangle$ provided for this fixed $t>0,\left\langle J_{i}(t), J_{j}(t)\right\rangle=\delta_{i j}$. 
Let $\widetilde{M}$ be hyperbolic space form with curvature $K_{\widetilde{M}}=-b^{2}$. Similarly, we have

$$
\frac{\tilde{\phi}^{\prime}(t)}{\tilde{\phi}(t)}=\left(\tilde{\Delta} \tilde{\rho}-\frac{n-1}{\tilde{\rho}}\right) \circ \tilde{\gamma}(t)
$$

Here, the meaning of the notation with tilde on its top is the same as that of the above notation without tilde. Thus, we obtain

$$
\frac{\phi^{\prime}(t)}{\phi(t)}-\frac{\widetilde{\phi}^{\prime}(t)}{\widetilde{\phi}(t)}=(\Delta \rho) \circ \gamma(t)-(\widetilde{\Delta} \tilde{\rho}) \circ \widetilde{\gamma}(t)
$$

Using theorem $\mathrm{C}$, we obtain

$$
\frac{\phi^{\prime}(t)}{\phi(t)}-\frac{\bar{\phi}^{\prime}(t)}{\tilde{\phi}(t)} \geqslant-\frac{n-2}{n-1}(\tilde{\Delta} \tilde{\rho}) \circ \tilde{\gamma}(t)=-(n-2) b \operatorname{coth}(b t) .
$$

Integrating the above inequality on $\left[t_{1}, t_{2}\right]$ with $t_{2}>t_{1}>0$, we have

$$
\ln \frac{\phi\left(t_{2}\right)}{\phi\left(t_{1}\right)}-\ln \frac{\tilde{\phi}\left(t_{2}\right)}{\tilde{\phi}\left(t_{1}\right)} \geqslant-(n-2) \ln \left(\frac{e^{b t_{2}}-e^{-b t_{2}}}{e^{b t_{1}}-e^{-b t_{1}}}\right) \text {. }
$$

Therefore, $\frac{\phi(t)}{\widetilde{\phi}(t)}\left(e^{b t}-e^{-b t}\right)^{n-2}$ is an increasing function. Let $S_{x_{0}}(r), \widetilde{S}_{\widetilde{x}_{0}}(r) \cdot$ be the geodesic spheres centred at $x_{0}, \widetilde{x}_{0}$ with radius $r$ in $M, \widetilde{M}$, respectively, and $A(r), \widetilde{A}(r)$ the derived Riemannian volume of $S_{x_{0}}(r), \widetilde{S}_{\widetilde{x}_{0}}(r)$ in $M, \widetilde{M}$, respectively. Then

$$
\left(e^{b r}-e^{-b r}\right)^{n-2} \frac{A(r)}{\widetilde{A}(r)}
$$

is increasing. Therefore,

$$
\frac{\int_{0}^{r}\left(e^{b s}-e^{-b s}\right)^{n-2} A(s) d s}{\int_{0}^{r} \widetilde{A}(s) d s}
$$

is also increasing. For any $\varepsilon>0$, when $r>\varepsilon$, we have

$$
\begin{aligned}
\int_{0}^{r}\left(e^{b s}-e^{-b s}\right)^{n-2} A(s) d s & \geqslant \frac{\int_{0}^{e}\left(e^{b s}-e^{-b s}\right)^{n-2} A(s) d s}{\int_{0}^{e} \widetilde{A}(s) d s} \int_{0}^{r} \widetilde{A}(s) d s \\
& =c(\varepsilon, n) \tilde{V}_{\tilde{x}_{0}}(r), \quad c(\varepsilon, n)>0 .
\end{aligned}
$$

So, we obtain

$$
e^{(n-2) b r} V_{x_{0}}(r) \geqslant c(\varepsilon, n) \tilde{V}_{\tilde{x}_{0}}(r), \quad r>\varepsilon .
$$

On the other hand, $\widetilde{V}_{\widetilde{x}_{0}}(r) \approx e^{(n-1) b r}$. Finally, we have

$$
V_{x_{0}}(r) \geqslant c^{\prime}(\varepsilon, n) e^{b r}, \quad r>\varepsilon .
$$

This completes the proof of Theorem B. 


\section{ReFERENCES}

[1] J. Milnor, 'A note on curvature and fundamental group', J. Differential Geom. 2 (1968), 1-7.

[2] Q. Ding, 'The Dirichlet Problem at infinity for manifolds of nonpositive curvature', in Differential Geometry Proceedings Symposium in honor of $S u$ Buchin on his $90^{\text {th }}$ birthday (World Sci. Publ. Co., 1993), pp. 49-58.

[3] Y.L. Xin, 'Harmonic maps of the bounded symmetric domains', Math. Ann. 303 (1995), 417-433.

Institute of Applied Mathematics,

Academia Sinica,

Beijing 100080,

Peoples Republic of China. 